\title{
Buckling of Doubly Clamped Nano-Actuators in General Form through Optimized Chebyshev Polynomials with Interior Point Algorithm
}

\begin{abstract}
E. Shivanian* AND M.R. Ansari
Department of Applied Mathematics, Imam Khomeini International University, Qazvin, 34149-16818, Iran

(Received August 21, 2018; in final form January 10, 2019)

In current work, a novel intelligent computational technique is adopted for searching the behaviour of the problem of buckling of nano-actuators in the presence of various nonlinear forces. In order to accomplish this aim, the governing integro-differential equation in general form is taken into account for nano-actuators, which contains various nonlinear forces as well. This generalized form for the nano-actuators is in fact a non-linear fourth-order Fredholm integro-differential boundary value problem. The boundary value problem is transformed into an equivalent problem whose boundary conditions are such that it is convenient to apply reformed version of the Chebyshev polynomials of the first kind. These Chebyshev polynomials based functions construct approximate series solution with unknown weights. The mathematical formulation of optimization problem consists of an uninspected error which is minimized through adjusting weights via interior point method. The trial approximate solution is validated by imposing tolerance constrained into optimization problem. This numerical technique enables us to overcome all kind of nonlinearities in the mentioned boundary value problem, and then to obtain an accurate solution. Thus, it can expedite the design of nano-actuators.
\end{abstract}

DOI: 10.12693/APhysPolA.135.444

PACS/topics: nano-actuator, Chebyshev polynomial of the first kind, interior point method, Fredholm integral equation

\section{Preliminaries and mathematical formulation}

Consider the following generalized form of the governing problem of a nano-actuator beam, augmented to boundary conditions, and containing the influence of axial loads and other various kind of nonlinear forces [1]:

$$
\begin{aligned}
& \frac{\mathrm{d}^{4} u}{\mathrm{~d} x^{4}}-\left(\eta \int_{0}^{1}\left(\frac{\mathrm{d} u}{\mathrm{~d} x}\right)^{2} \mathrm{~d} x+P\right) \frac{\mathrm{d}^{2} u}{\mathrm{~d} x^{2}}+\frac{\alpha}{u^{\zeta}} \\
& +\frac{\beta}{(\kappa+u)^{2}}+\frac{\gamma}{u}=0, \quad x \in[0,1], \\
& u(0)=u(1)=1, \quad u^{\prime}(0)=u^{\prime}(1)=0,
\end{aligned}
$$

where all variables and parameters are in the nondimensional form, with $u$ and $x$ being deflection and the length of the beam, respectively. $P$ and $\eta$ denote the influence of axial forces, $\beta$ indicates the influence of the external imposed voltage, $\kappa$ denotes the influence of a dielectric layer, $\gamma$ indicates capillary impact or the fringing field, $\zeta$ is an integer positive number and the concept of the parameter $\alpha$ depends on the value of $\zeta$ e.g. in the case of $\zeta=3, \alpha$ represents the van der Waals impacts, and in the case of $\zeta=4, \alpha$ represents the Casimir impact.

In fact, an essential and unavoidable portion of a large number of nano/micro electromechanical systems is a nano/micro actuator. The actuator contains a beam

*corresponding author; e-mail: shivanian@sci.ikiu.ac.ir hung over a substratum in nano/micro mechanical actuators, or in nano/micro mechanical sensors. Electrostatic field is induced by imposing a voltage difference among the substrate and beam, and therefore, the electrostatic forces captivate the beam into the substrate [1]. Furthermore, beam and substrate play the role of a condenser, where the movement of the beam could be discerned by the capacitive alteration as a signal [2]. The nano-actuators are in the presence of various inherently nonlinear forces such as dielectric effects, fringing field effects, the van der Waals attractions, and the Casimir force $[3-5]$. On the other hand, the axial forces in the clamped-clamped kind of nano-actuators is consequential matter, and should be considered. The subject of a similar kind of axial forces causes an integro-differential expression to be generated in the governing equation of the nano-actuators [6-12]. As a conclusion, since the actuators are constructed in billions for chipsets as well as in thousands for sensors in the practical applications of nano-/micro-actuators then, presenting more accurate technique in order to analyze nano/micro structures including these actuators is of more interest to the researchers.

The boundary value problem (BVP) (1), (2) has been studied in some special cases. In Ref. [7] authors considered term including $P$. Authors of $[3,7,8]$ studied the force $\eta \int_{0}^{1}\left(\frac{\mathrm{d} u}{\mathrm{~d} x}\right)^{2} \mathrm{~d} x$. In Refs. [13, 14], the term containing $\beta$ is studied. Authors in [14-16] considered the force $\gamma$. Also, the nonlinear forces term $\frac{\alpha}{u^{3}}$ are considered in $[4,15]$, the nonlinear forces terms $\frac{\alpha}{u^{4}}$ are considered in $[5,16,17]$ and $\kappa$ is considered in [3]. 
The BVP (1), (2) in the general form has been analyzed by a modification of Adomian decomposition method $(\mathrm{ADM})$, more specifically Duan-Rach Adomian decomposition method [1]. ADM and its modifications are some kinds of semi-analytical methods [18-20]. It has been suggested by many researchers that ADM is not often reliable, and it does have many shortcomings especially when it comes to the subject of convergence of the solution. According to Jiao et al. [21], "although the series can be rapidly convergent in a very small region, it has very slow convergence rate in the wider region and the truncated series solution is an inaccurate solution in that region, which will greatly restrict the application area of the method". Moreover, the BVP (1), (2) has been analyzed by meshless method through radial basis functions, though they are often used for high-dimensional problems [22-35]. The interested readers are also referred to [36-39] for other useful techniques, which can be applied for the same problems.

In this article, we present a novel intelligent computational technique to obtain solution for non-linear fourthorder Fredholm integro-differential BVP (1), (2). First, we transform the governing equation into an equivalent problem whose boundary conditions are $[-1,1]$. Now, it is convenient to apply reformed version of the Chebyshev polynomials of the first kind. Then, we optimize the Chebyshev polynomials of the first kind to construct approximate series solution with unknown weights. Furthermore, an optimization problem is constructed [9] based on unsupervised error as objective function in the presence of a tolerance as restriction. This optimization problem is minimized by tuning weights via interior point method. This numerical based technique enables us to overcome all kind of nonlinearities in the mentioned BVP and then to obtain accurate solution. Therefore, it enables us to expedite the design of nano-actuators. Moreover, in some easy linear cases, we compare the approximate solution obtained with the exact one.

\section{High-order derivatives of basis functions}

The Chebyshev orthogonal polynomials on the interval $[-1,1][40]$ are particularly useful. These polynomials do have many good characteristic in the performance of approximating functions, and so they are used regularly in many fields of applied mathematics, physics and engineering.

\subsection{Basic properties of the Chebyshev polynomials}

The Chebyshev orthogonal polynomials of the first kind, known as $T_{n}(x)=\cos (n \arccos x)$, can be calculated via Rodrigue's expression as [41]:

$$
\begin{aligned}
& T_{n}(x)=\frac{\Gamma\left(\frac{1}{2}\right)}{(-2)^{n} \Gamma\left(n+\frac{1}{2}\right)} \sqrt{1-x^{2}} \frac{\mathrm{d}^{n}}{\mathrm{~d} x^{n}}\left(1-x^{2}\right)^{n-\frac{1}{2}}, \\
& n=0,1,2, \ldots
\end{aligned}
$$

The Chebyshev orthogonal polynomials of the first kind could be also obtained through the generating function, as follows:

$$
\frac{1-t x}{1-2 t x+t^{2}}=\sum_{n=0}^{+\infty} T_{n}(x) t^{n} .
$$

The first and the second Chebyshev orthogonal polynomials i.e. $T_{1}(x)=1$ and $T_{2}(x)=x$ are obviously obtained from (3), and all other polynomials $T_{n}(x), n \geq 2$ are given by the well known recursion relation

$$
T_{n+1}(x)=2 x T_{n}(x)-T_{n-1}(x) .
$$

Also, the derivative of $T_{n}(x)$ with respect to $x$ could be easily given by

$$
\begin{aligned}
& \left(1-x^{2}\right) T_{n}^{\prime}(x)=-n x T_{n}(x)+n T_{n-1}(x), x \neq \pm 1, \\
& T_{n}^{\prime}(-1)=n^{2}(-1)^{n+1}, \quad T_{n}^{\prime}(1)=n^{2} .
\end{aligned}
$$

The following characteristics of $T_{n}(x)$ with some special values are well established, and will be useful in the following sections:

$$
\begin{aligned}
& T_{n}(-x)=(-1)^{n} T_{n}(x), T_{n}(1)=1, T_{n}(-1)=(-1)^{n}, \\
& T_{2 n}(0)=(-1)^{n}, \quad T_{2 n+1}(0)=0 .
\end{aligned}
$$

Moreover, the orthogonality characteristics of the Chebyshev polynomials is the direct results of our understanding of the orthogonality of the cosine functions, i.e.

$$
\int_{-1}^{1} \frac{T_{n}(x) T_{m}(x)}{\sqrt{1-x^{2}}} \mathrm{~d} x= \begin{cases}0, & m \neq n, \\ \frac{\pi}{2}, & m=n \neq 0, \\ \pi, & m=n=0 .\end{cases}
$$

It is seen that the set of the Chebyshev polynomials constructs an orthogonal system on the interval $[-1,1]$ augmented to the weight function $\frac{1}{\sqrt{1-x^{2}}}$.

\subsection{High-order derivatives of the Chebyshev polynomials}

(The Leibniz formula) Considering the function $f(x)=g(x) h(x)$, the $k$-th derivative of $f(x)$ could be expanded as a sum of derivatives of $g(x)$ and $h(x)$ as follows:

$$
f^{(k)}(x)=\sum_{n=0}^{k}\left(\begin{array}{l}
k \\
n
\end{array}\right) g^{(n)}(x) h^{(k-n)}(x),
$$

in which $\left(\begin{array}{l}k \\ n\end{array}\right)$ are the binomial coefficients.

\section{Theorem 2.1 (Slevinsky-Safouhi) [42]}

Let $G(x)$ be a function $k$-th differentiable and with the term $\left(\frac{\mathrm{d}}{x \mathrm{~d} x}\right)^{k} G(x)$ well defined. The term $\frac{\mathrm{d}^{k} G}{\mathrm{~d} x^{k}}$ can be expressed by

$$
\frac{\mathrm{d}^{k} G}{\mathrm{~d} x^{k}}=\sum_{i=\left\lfloor\frac{k+1}{2}\right\rfloor}^{k} \hat{A}_{k}^{i} x^{2 i-k}\left(\frac{\mathrm{d}}{x \mathrm{~d} x}\right)^{k} G(x),
$$

with coefficients

$\hat{A}_{k}^{i}=$

$$
\begin{cases}1, & i=k ; \\ 2 \hat{A}_{k-1}^{i}+\hat{A}_{k-1}^{i-1}, & i=\left\lfloor\frac{k+1}{2}\right\rfloor, k \text { odd, } \\ \hat{A}_{k-1}^{i}, & i=\left\lfloor\frac{k+1}{2}\right\rfloor, k \text { even, } \\ (2 i-k+1) \hat{A}_{k-1}^{i}+\hat{A}_{k-1}^{i-1}, & \left\lfloor\frac{k+1}{2}\right\rfloor<i<k, k>3 .\end{cases}
$$

where $\lfloor\alpha\rfloor$ is the integer floor function of argument $\alpha$. 
It is not difficult to make use of the Leibniz formula as well as Rodrigue's formula to obtain the higher order derivatives of $T_{n}(x)$ as

$$
\frac{\mathrm{d}^{i}}{\mathrm{~d} x^{i}} T_{n}(x)=\frac{\Gamma\left(\frac{1}{2}\right)}{(-2)^{n} \Gamma\left(n+\frac{1}{2}\right)}
$$

$$
\times \sum_{l=0}^{i}\left(\begin{array}{l}
i \\
l
\end{array}\right) \frac{\mathrm{d}^{l}}{\mathrm{~d} x^{l}} \sqrt{1-x^{2}} \frac{\mathrm{d}^{n+i-l}}{\mathrm{~d} x^{n+i-l}}\left(1-x^{2}\right)^{n-\frac{1}{2}} .
$$

Without going into minute details, if we apply the result of Theorem 2.1 into the above equation, then we develop a very effective formula as the final result [42]:

$$
\begin{aligned}
& \frac{\mathrm{d}^{k}}{\mathrm{~d} x^{k}} T_{n}(x)=\frac{\Gamma\left(\frac{1}{2}\right)}{(-2)^{n} \Gamma\left(n+\frac{1}{2}\right)} \sum_{l=0}^{k}\left\{\left(\begin{array}{l}
k \\
l
\end{array}\right)\left[\sum_{i=\left\lfloor\frac{l+1}{2}\right\rfloor}^{l} \hat{A}_{l}^{i} x^{2 i-l}(-2)^{i}\left(1-x^{2}\right)^{\frac{1}{2}-i} \prod_{j=0}^{i-1}\left(\frac{1}{2}-j\right)\right]\right. \\
& \left.\quad \times\left[\sum_{i=\left\lfloor\frac{n+k-l+1}{2}\right\rfloor}^{n+k-l} \hat{A}_{n+k-l}^{i} x^{2 i-n-k+l}(-2)^{i}\left(1-x^{2}\right)^{n-\frac{1}{2}-i} \prod_{j=0}^{i-1}\left(n-\frac{1}{2}-j\right)\right]\right\}
\end{aligned}
$$

with coefficients $\hat{A}_{k}^{i}$ given by (12).

\section{Proposed method}

By changing the variable $x \mapsto \frac{1}{2}(x+1)$, the boundary value problem (1),(2) can be rewritten as

$$
\begin{aligned}
& 16 \frac{\mathrm{d}^{4} u}{\mathrm{~d} x^{4}}-4\left(2 \eta \int_{-1}^{1}\left(\frac{\mathrm{d} u}{\mathrm{~d} x}\right)^{2} \mathrm{~d} x+P\right) \frac{\mathrm{d}^{2} u}{\mathrm{~d} x^{2}} \\
& \quad+\frac{\alpha}{u^{\zeta}}+\frac{\beta}{(\kappa+u)^{2}}+\frac{\gamma}{u}=0, \quad x \in[-1,1], \\
& u(-1)=u(1)=1, u^{\prime}(-1)=u^{\prime}(1)=0 .
\end{aligned}
$$

Now, it is convenient to treat the Chebyshev polynomials of the first kind. In addition, the integral term can also be evaluated by the Gauss-Legendre quadrature. Moreover, the change of function $u \mapsto u+1$ transforms the problem into

$$
\begin{gathered}
16 \frac{\mathrm{d}^{4} u}{\mathrm{~d} x^{4}}-4\left(2 \eta \int_{-1}^{1}\left(\frac{\mathrm{d} u}{\mathrm{~d} x}\right)^{2} \mathrm{~d} x+P\right) \frac{\mathrm{d}^{2} u}{\mathrm{~d} x^{2}} \\
+\frac{\alpha}{(u+1)^{\zeta}}+\frac{\beta}{(\kappa+u+1)^{2}}+\frac{\gamma}{u+1}=0, \\
u(-1)=u(1)=0, u^{\prime}(-1)=u^{\prime}(1)=0,
\end{gathered}
$$

such that the boundary condition becomes homogeneous.

3.1. Reformed version of the Chebyshev polynomials

Define $\hat{T}_{n}, n \geq 1$ as

$$
\hat{T}_{n}(x)=T_{2 n}(x)-2 n^{2} x^{2}-1+2 n^{2}, \quad n \geq 1,
$$

then obviously, from (8), we have

$$
\hat{T}_{n}(-1)=\hat{T}_{n}(1)=0, \quad n \geq 1 .
$$

Equation (7) implies

$$
\begin{aligned}
& \hat{T}_{n}^{\prime}(-1)=T_{2 n}^{\prime}(-1)+4 n^{2}=-4 n^{2}+4 n^{2}=0, \\
& n \geq 1, \\
& \hat{T}_{n}^{\prime}(1)=T_{2 n}^{\prime}(1)-4 n^{2}=4 n^{2}-4 n^{2}=0 \quad n \geq 1 .
\end{aligned}
$$

Therefore, from Eqs. (20)-(22), we conclude that the boundary conditions (18) hold.
Furthermore, the second and forth derivative of reformed version of the Chebyshev polynomials of the first kind are given by

$$
\begin{aligned}
& \hat{T}_{n}^{\prime}(x)=T_{2 n}^{\prime}(x)-4 n^{2} x, \quad n \geq 1, \\
& \hat{T}_{n}^{\prime \prime}(x)=T_{2 n}^{\prime \prime}(x)-4 n^{2}, \quad n \geq 1, \\
& \hat{T}_{n}^{\prime \prime \prime \prime}(x)=T_{2 n}^{\prime \prime \prime \prime}(x), \quad n \geq 1,
\end{aligned}
$$

where the right hand side can be obtained by Eq. (14) when $k=1,2,4$.

\subsection{Corresponding optimization problem}

We define a approximate series solution of order $M$ as

$$
U_{M}(x)=\sum_{n=1}^{M} \alpha_{n} \hat{T}_{n}(x),
$$

and we consider the number of $N$ regularly distributed nodal points in interval $[-1,1]$, namely $x_{i}, i=1,2, \ldots, N$. Then we define the uninspected error as the sum of mean squared errors

$$
\begin{aligned}
& \epsilon(N, \alpha)=\frac{1}{N} \sum_{i=1}^{N}\left\{16 \sum_{n=1}^{M} \alpha_{n} \hat{T}_{n}^{\prime \prime \prime \prime}\left(x_{i}\right)\right. \\
& -4\left[2 \eta \int_{-1}^{1}\left(\sum_{n=1}^{M} \alpha_{n} \hat{T}_{n}^{\prime}(x)\right)^{2} \mathrm{~d} x+P\right] \sum_{n=1}^{M} \alpha_{n} \hat{T}_{n}^{\prime \prime}\left(x_{i}\right) \\
& +\frac{\alpha}{\left(\sum_{n=1}^{M} \alpha_{n} \hat{T}_{n}\left(x_{i}\right)+1\right)^{\zeta}}+\frac{\beta}{\left(\kappa+\sum_{n=1}^{M} \alpha_{n} \hat{T}_{n}\left(x_{i}\right)+1\right)^{2}} \\
& \left.+\frac{\gamma}{\sum_{n=1}^{M} \alpha_{n} \hat{T}_{n}\left(x_{i}\right)+1}\right\}^{2}
\end{aligned}
$$


where the term $\int_{-1}^{1}\left(\sum_{n=1}^{M} \alpha_{n} \hat{T}_{n}^{\prime}(x)\right)^{2} \mathrm{~d} x$ is properly evaluated by the powerful Gauss-Legendre quadrature formula. It is worth to mention here that $U_{M}(x)$ automatically satisfy all boundary conditions (18). Now, we define the following optimization problem:

$$
\min _{\alpha} \epsilon(N, \alpha) \text { subject to } \epsilon(N, \alpha)-\varepsilon \leq 0,
$$

where $\varepsilon$ is a given tolerance. In our approach, the interior point method (IPM) is used for tuning of weights of the approximate series solution (26). IPM belongs to a class of algorithms which are useful tools in treating constrained optimization problems. The technique is based upon Karmarkar's algorithm which has been improved by Narendra Karmarkar in 1984 for linear programming resolution [43]. Detailed in formation about the algorithm can be observed in Refs. [44, 45]. IPMs have been applied to many optimization problems in engineering and applied science such as multi-area optimal reactive power flow [46] and economic dispatch problem [47]. The fundamental trait of interior point methods are established upon self-concordant barrier functions which play important role in encoding the convex set. As opposed to the classical simplex method, searching for an optimum solution is done by traversing the interior of the feasible domain, and solving a sequence of some sub-problems [48].

\section{Numerical experiments and comparison}

In current section, we present the results obtained for some case studies, which have been adopted from Ref. [1], using proposed method described in the previous sections. In these examples, the number of total nodal points covering $[-1,1], N=20$, and they are regularly distributed. Also, the integral term in Eq. (27) is evaluated with 8-points Gaussian quadrature rule. Moreover, the number of basis function in approximate series solution in Eq. (26) is $M=6$. The obtained solutions can be compared to those of Ref. [1] and references therein. All approximate solutions reported here are obtained, in few seconds by MATLAB software programme, and therefore the method is highly robust.

MATLAB provides an efficient optimization toolbox that contains functions for finding minimum of a multivariable function while satisfying constraints. The toolbox includes solvers that perform optimization on the various types of linear or nonlinear problems. The function, fmincon $(\cdot)$, of this toolbox is a general, multipurpose optimizer that is well tested and frequently used to solve nonlinear programming problems with general equality, inequality, and bound constraints of small, medium, and large scale. To handle optimization problem (28), we use fmincon(.) augmented to the IPM as described in the previous section.

As the first case study, we consider $P=\alpha=\kappa=\gamma=$ $0, \eta=10$. The maximum deflection i.e. $1-u(0.5)$ versus the parameter $\beta$ has been plotted in Fig. 1. In the same case, some approximate solutions (i.e. deflection of beam versus the length of beam) have been shown in

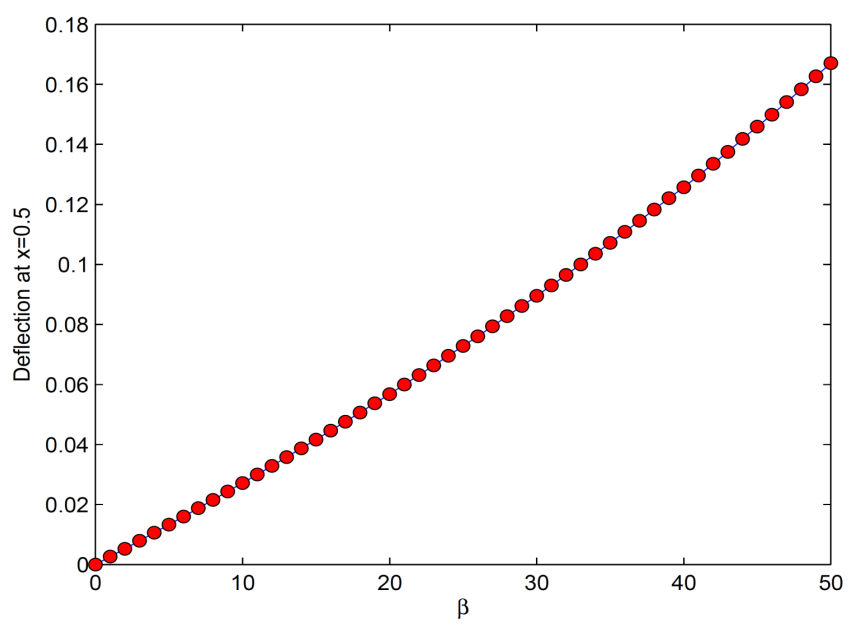

Fig. 1. The maximum deflection via $\beta$ with $P=\alpha=$ $\kappa=\gamma=0, \eta=10$.

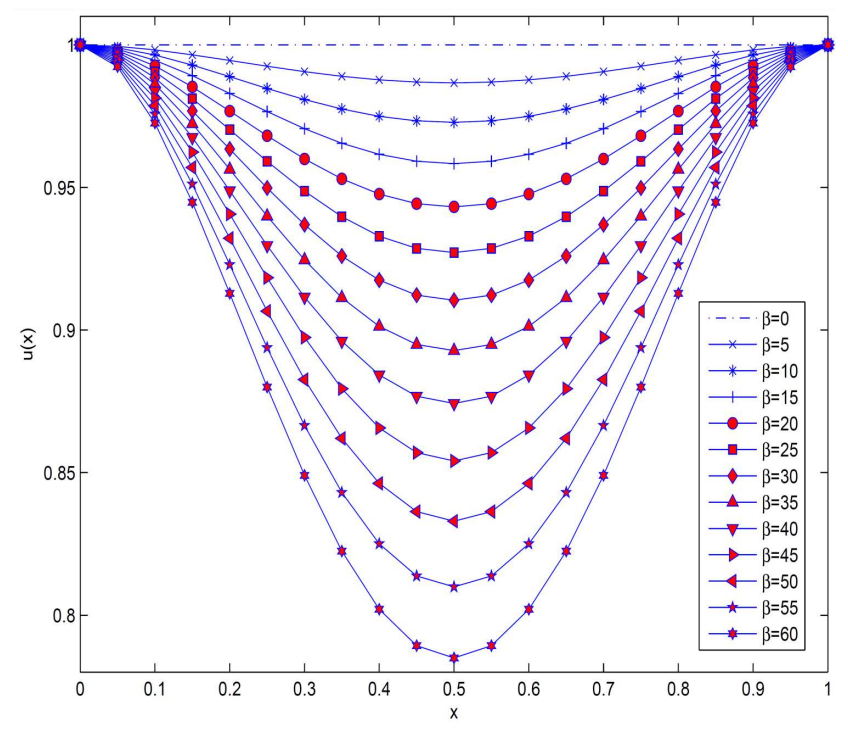

Fig. 2. The approximate solutions for different $\beta$ with $P=\alpha=\kappa=\gamma=0, \eta=10$.

Fig. 2. The approximate solution, from (26), are given below for some $\beta \mathrm{s}$ in this case

$$
\begin{aligned}
& U_{6}(x)=0 \hat{T}_{1}(x)+0 \hat{T}_{2}(x)-0.001663652712040 \hat{T}_{3}(x) \\
& \quad+0.000000900926945 \hat{T}_{4}(x) \\
& \quad-0.000000006756785 \hat{T}_{5}(x) \\
& \quad-0.000000001099784 \hat{T}_{6}(x), \quad \beta=5, \\
& U_{6}(x)=0 \hat{T}_{1}(x)+0 \hat{T}_{2}(x)-0.011275420680401 \hat{T}_{3}(x) \\
& \quad+0.000045843324410 \hat{T}_{4}(x) \\
& \quad-0.000002215204480 \hat{T}_{5}(x) \quad \\
& +0.000000025243543 \hat{T}_{6}(x), \quad \beta=30,
\end{aligned}
$$




$$
\begin{aligned}
& U_{6}(x)=0 \hat{T}_{1}(x)+0 \hat{T}_{2}(x)-0.027262066428867 \hat{T}_{3}(x) \\
& \quad+0.0002244448017465 \hat{T}_{4}(x) \\
& \quad-0.000017716166222 \hat{T}_{5}(x) \\
& +0.000000557044021 \hat{T}_{6}(x), \quad \beta=60,
\end{aligned}
$$

where $\hat{T}_{n}(x)$ is given by Eq. (19). For the second case study, we assume $P=\alpha=\kappa=\beta=0, \eta=10$, and the maximum deflection versus the parameter $\gamma$ has been plotted in Fig. 3. For the third case study, we consider $P=\gamma=\kappa=\beta=0, \zeta=3, \eta=10$, and the maximum deflection versus the parameter $\alpha$ has been shown in Fig. 4 . For the forth case study, we suppose $P=0, \alpha=20$, $\kappa=-0.396, \gamma=0.325, \zeta=4, \beta=5$ and $\eta=0.96$, the approximate solution is seen in Fig. 5. For the last case study, we set $P=5, \alpha=1, \kappa=1, \gamma=1, \zeta=2$, and $\eta=10$, and some approximate solutions (i.e. deflection of beam versus the length of beam) have been shown in Fig. 6 for some different $\beta$.

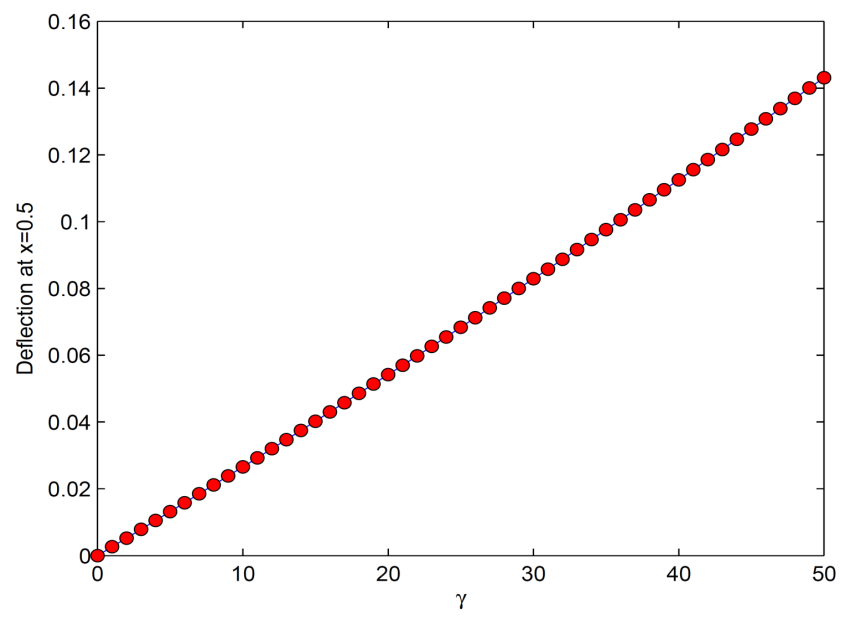

Fig. 3. The maximum deflection via $\gamma$ with $P=\alpha=$ $\kappa=\beta=0, \eta=10$

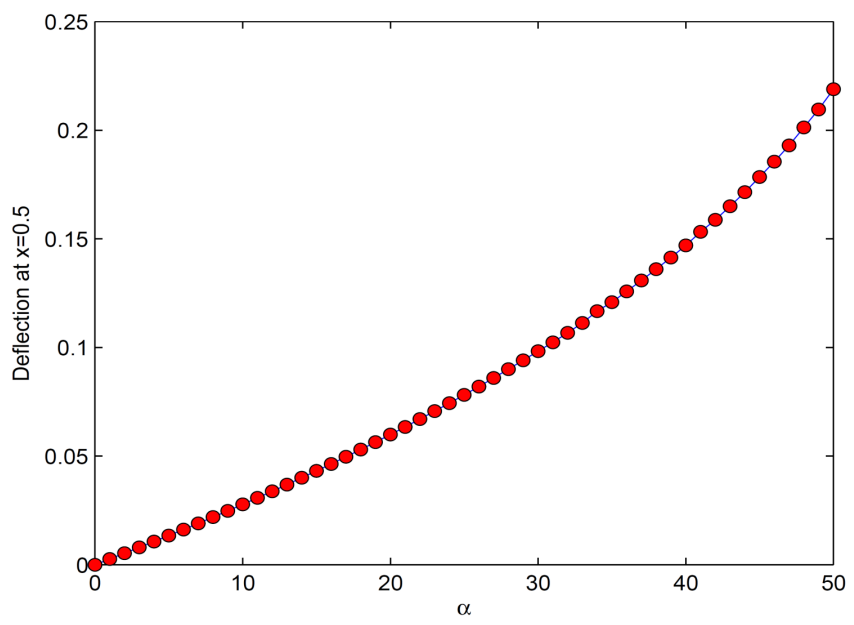

Fig. 4. The maximum deflection via $\alpha$ with $P=\gamma=$ $\kappa=\beta=0, \zeta=3, \eta=10$.

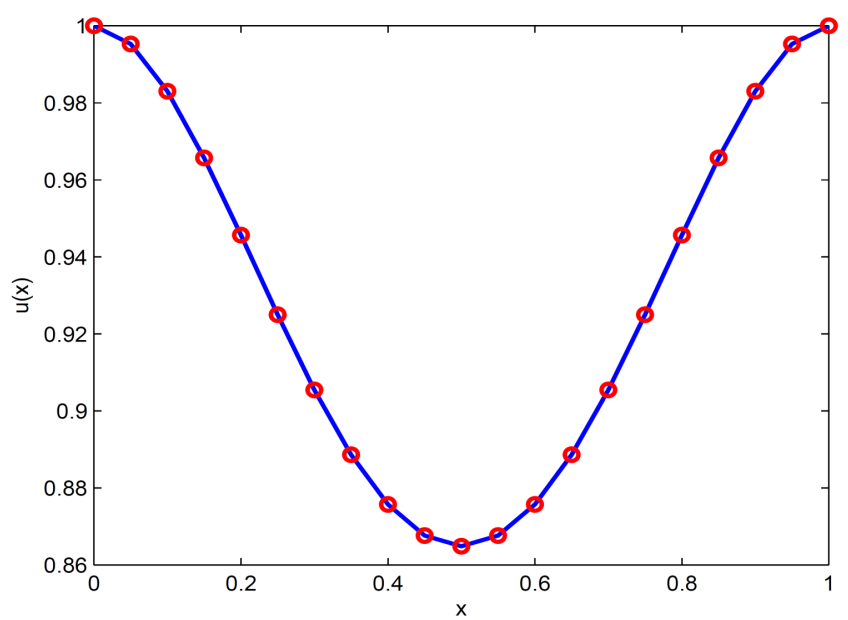

Fig. 5. The approximate solutions with $P=0, \alpha=20$, $\kappa=-0.396, \gamma=0.325, \zeta=4, \beta=5$ and $\eta=0.96$.

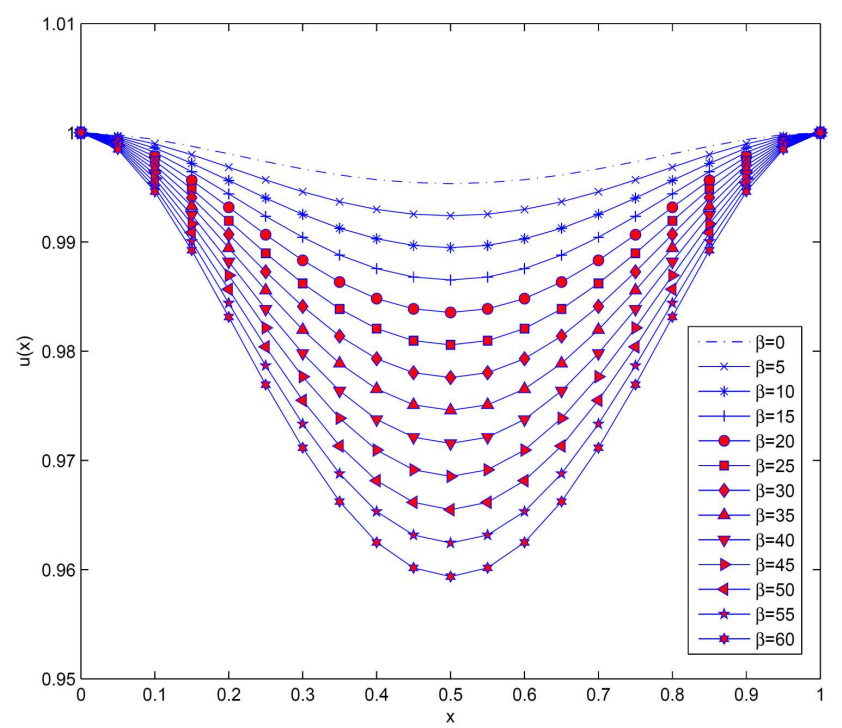

Fig. 6. The approximate solutions for different $\beta$ with $P=5, \alpha=1, \kappa=1, \gamma=1, \zeta=2$, and $\eta=10$.

To validate our results, we consider the boundary value problem (1),(2) and set $\alpha=\beta=\gamma=0$. Then it leads to

$$
\begin{aligned}
& \frac{\mathrm{d}^{4} u}{\mathrm{~d} x^{4}}-\left(\eta \int_{0}^{1}\left(\frac{\mathrm{d} u}{\mathrm{~d} x}\right)^{2} \mathrm{~d} x+P\right) \frac{\mathrm{d}^{2} u}{\mathrm{~d} x^{2}}=0, \\
& x \in[0,1], \\
& u(0)=u(1)=1, u^{\prime}(0)=u^{\prime}(1)=0 .
\end{aligned}
$$

It is easy to see that the unique solution to the above equations is $u(x) \equiv 1$. This point is in full agreement with our approximation result shown in Fig. 2 when $\beta=0$. In the case where the parameters are not zero, our results are comparable to those results obtained by $\mathrm{ADM}$ in [1]. 


\section{Conclusions}

The differential equation governing clamped-clamped nano-actuators has been considered in a generalized sense, which the nano-actuators are in the presence of various nonlinear forces, like the van der Waals force, the Casimir force, applied voltage, fringing field effect, capillary effect, and the dielectric layer effect. Moreover, there is a nonlinear integral expression in the governing problem of the nano-actuator because of the presence of the axial loads, which makes it difficult to be solved. We propose a new intelligent computational technique to obtain approximate solution for the mentioned problem. First, the governing equation is transformed into an equivalent problem whose boundary conditions are homogeneous in interval $[-1,1]$. Then, optimized Chebyshev polynomials of the first kind are used to construct approximate series solution with unknown weights. Furthermore, by defining an optimization problem and minimizing it, all weights are obtained via interior point method. It has been revealed through test studies that the method is highly robust and reliable.

\section{Acknowledgments}

The authors are grateful to anonymous reviewers for carefully reading this paper and for their valuable comments and suggestions, which have improved the paper.

\section{References}

[1] M. Ghalambaz, M. Ghalambaz, M. Edalatifar, Appl. Math. Modell. 40, 7293 (2016).

[2] R. Ansari, R. Gholami, M.F. Shojaei, V. Mohammadi, S. Sahmani, Acta Astronaut. 102, 140 (2014).

[3] E. Yazdanpanahi, A. Noghrehabadi, M. Ghalambaz, Int. J. Non-Lin. Mech. 58, 128 (2014).

[4] R. Soroush, A. Koochi, A.S. Kazemi, M. Abadyan, Int. J. Struct. Stabil. Dynam. 12, 1250036 (2012).

[5] A. Koochi, A.S. Kazemi, Y.T. Beni, A. Yekrangi, Physica E Low-Dim. Syst. Nanostruct. 43, 625 (2010).

[6] B. Abbasnejad, G. Rezazadeh, R. Shabani, Acta Mech. Solida Sin. 26, 427 (2013).

[7] E.M. Abdel-Rahman, M.I. Younis, A.H. Nayfeh, J. Micromech. Microeng. 12, 759 (2002).

[8] B. Choi, E. Lovell, J. Micromech. Microeng. 7, 24 (1997).

[9] A. Noghrehabadi, M. Ghalambaz, A. Vosough, Int. J. Multidiscipl. Sci. Eng. 2, 22 (2011).

[10] M. Ghalambaz, A. Noghrehabadi, A. Vosoogh, Int. Rev. Mech. Eng. 5, 586 (2011).

[11] A. Noghrehabadi, M. Ghalambaz, Y.T. Beni, M. Abadyan, M.N. Abadi, M.N. Abadi, Proced. Eng. 10, 3725 (2011).

[12] M. Ghalambaz, A. Noghrehabadi, M. Abadyan, Y.T. Beni, A.R.N. Abadi, M.N. Abadi, Proced. Eng. 10, 3708 (2011).
[13] J.-H. Kuang, C.-J. Chen, Math. Comput. Modell. 41, 1479 (2005).

[14] Y. Gerson, I. Sokolov, T. Nachmias, B. Ilic, S. Lulinsky, S. Krylov, Sens. Actuat. A Phys. 199, 227 (2013).

[15] A. Koochi, H. Hosseini-Toudeshky, H.R. Ovesy, M. Abadyan, Int. J. Struct. Stabil. Dynam. 13, 1250072 (2013).

[16] A. Noghrehabadi, M. Ghalambaz, A. Ghanbarzadeh, Comput. Math. Appl. 64, 2806 (2012).

[17] A. Koochi, A. Kazemi, F. Khandani, M. Abadyan, Phys. Scr. 85, 035804 (2012).

[18] G. Adomian, Solving Frontier Problems of Physics: The Decomposition Method, Vol. 60, Springer Science \& Business Media, 2013.

[19] S. Liao, Homotopy Analysis Method in Nonlinear Differential Equations, Springer, 2012.

[20] A.-M. Wazwaz, Appl. Math. Computat. 161, 543 (2005).

[21] Y. Jiao, Y. Yamamoto, C. Dang, Y. Hao, Comput. Math. Appl. 43, 783 (2002).

[22] E. Shivanian, H.R. Khodabandehlo, Ain Shams Eng. J. 7, 993 (2016).

[23] E. Shivanian, Eng. Anal. Boundary Elem. 37, 1693 (2013).

[24] E. Shivanian, Eng. Anal. Boundary Elem. 50, 249 (2015).

[25] A. Shirzadi, F. Takhtabnoos, Inverse Probl. Sci. Eng. 24, 729 (2016).

[26] E. Shivanian, S. Abbasbandy, M.S. Alhuthali, H.H. Alsulami, Eng. Anal. Boundary Elem. 56, 98 (2015).

[27] V.R. Hosseini, E. Shivanian, W. Chen, Europ. Phys. J. Plus 130, 1 (2015).

[28] M. Aslefallah, E. Shivanian, Europ. Phys. J. Plus 130, 1 (2015).

[29] E. Shivanian, Int. J. Numer. Meth. Eng. 105, 83 (2016).

[30] S. Abbasbandy, H.R. Ghehsareh, I. Hashim, A. Alsaedi, Eng. Anal. Boundary Elem. 47, 10 (2014).

[31] V.R. Hosseini, W. Chen, Z. Avazzadeh, Eng. Anal. Boundary Elem. 38, 31 (2014).

[32] V.R. Hosseini, E. Shivanian, W. Chen, J. Computat. Phys. 312, 307 (2016).

[33] Z. Avazzadeh, V. Hosseini, W. Chen, Iran. J. Sci. Technol. (Sci.) 38, 205 (2014).

[34] E. Shivanian, Eng. Anal. Boundary Elem. 54, 1 (2015).

[35] E. Shivanian, A. Jafarabadi, Eng. Anal. Boundary Elem. 72, 42 (2016).

[36] E. Yazdanpanahi, A. Noghrehabadi, M. Ghalambaz, J. Mech. 30, 49 (2014).

[37] E. Yazdanpanahi, A. Noghrehabadi, M. Ghalambaz, Int. J. Mech. Sci. 74, 83 (2013).

[38] A. Noghrehabadi, M. Eslami, M. Ghalambaz, Int. J. Non-Lin. Mech. 52, 73 (2013).

[39] M. Ghalambaz, M. Ghalambaz, M. Edalatifar, J. Appl. Computat. Mech. 1, 207 (2015). 
[40] T. Rivlin, Chebyshev Polynomials, Wiley, New York 1975.

[41] N.H. Asmar, Partial Differential Equations with Fourier Series and Boundary Value Problems, Courier Dover Publ., 2016.

[42] R.M. Slevinsky, H. Safouhi, J. Computat. Appl. Math. 233, 405 (2009).

[43] N. Karmarkar, in: Proc. 16th Annual ACM Symposium on Theory of Computing, ACM, 1984, p. 302.

[44] S.J. Wright, Primal-Dual Interior-Point Methods, SIAM, 1997.
[45] M. Wright, Bull. Am. Math. Soc. 42, 39 (2005).

[46] W. Yan, L. Wen, W. Li, C. Chung, K. Wong, Int. J. Electr. Power Energy Syst. 33, 55 (2011).

[47] N. Duvvuru, K. Swarup, IEEE Trans. Power Syst. 26, 541 (2011).

[48] M.A.Z. Raja, R. Samar, Neurocomputing 124, 178 (2014). 